\title{
SEROPREVALENCIA DE Neospora caninum EN BOVINOS LECHEROS CRIADOS AL PASTOREO EN LA PROVINCIA DE MELGAR, PUNO
}

\author{
José Atoccsa H. ${ }^{1}$, Amanda Chávez V. ${ }^{2.3}$, Eva Casas A. ${ }^{2}$ y Néstor Falcón P. ${ }^{4}$
}

\section{Abstract}

The aim of the current study was to assess the seroprevalence of Neospora caninum in grazing dairy cattle in the province of Melgar, Puno, through serum antibody detection using the immunofluorescence indirect technique (IFI). A total of 419 sera was obtained from seven herds. The seroprevalence varied from $4.0 \pm 7.7 \%$ to $37.5 \pm 11.9 \%$, while the overall prevalence was $18.1 \pm 3.7 \%$, which was considered moderate. All herds showed at least one positive animal. Age and location showed to be a risk factor in the prevalence of the infection.

Key words: dairy cattle, Neospora caninum, IFI, neospororis, abortion

\section{RESUMEN}

El objetivo del presente estudio fue establecer la seroprevalencia de Neospora caninum en vacunos lecheros criados al pastoreo de la provincia de Melgar, Puno, mediante la detección de anticuerpos séricos por la técnica de inmunofluorescencia indirecta (IFI). Se evaluaron 419 sueros obtenidos en forma aleatoria de siete fundos ganaderos donde las prevalencias obtenidas variaron desde $4.0 \pm 7.7 \%$ hasta $37.5 \pm$ $11.9 \%$. La prevalencia general fue considerada moderada $(18.1 \pm 3.7 \%)$. Todos los fundos presentaron, al menos, un animal seropositivo. La edad y el lugar de procedencia representaron factores de riesgo en la prevalencia de la infección.

Palabras clave: bovino lechero, Neospora caninum, IFI, neosporosis, aborto

\author{
${ }^{1}$ Práctica privada \\ ${ }^{2}$ Laboratorio de Microbiología y Parasitología Veterinaria, FMV-UNMSM \\ ${ }^{3}$ E-mail: a_chavez_g@hotmail.com \\ ${ }^{4}$ Laboratorio de Medicina Veterinaria Preventiva, FMV-UNMSM
}




\section{INTRODUCCIÓN}

Los problemas infecciosos que interrumpen la gestación ocasionan cuantiosas pérdidas en los hatos lecheros (Rivera, 2001). Sólo en California, Estados Unidos, las pérdidas económicas asociadas con el aborto bovino por neosporosis se estiman en 35 millones de dólares anuales (Anderson et al., 1991; Barr et al., 1998).

La neosporosis ha sido descrita hace poco más de una década y, en la actualidad es considerada como una de las principales causas de aborto y mortalidad neonatal en el ganado vacuno lechero en diversos países (Dubey, 2003), siendo el agente causal el parásito Neospora caninum (Thurmond y Hietala, 1999). El papel de este agente etiológico como causal de abortos en el ganado vacuno es de suma importancia (Wouda et al., 2000), ya que puede presentarse de forma epidémica o endémica en un hato (Conraths y Schares, 1999).

Una característica importante de esta enfermedad en el vacuno es que el parásito puede permanecer latente como una infección crónica, de allí que la transmisión vertical o transplacentaria sea un elemento crucial en el establecimiento y la diseminación de la infección. Si la infección del feto no resulta en aborto, la cría resultante puede convertirse en portador clínicamente sano (asintomático) pero que podrá transmitir la infección a las siguientes generaciones (Anderson et al., 2000).

Estudios recientes indican que el $N$. caninum se viene convertiendo en un agente parasitario de gran importancia en el Perú, según los estudios de prevalencia realizados en las diversas cuencas lecheras (Rivera, 2001). En el país, la mayoría de investigaciones relacionadas a seroprevalencia de $N$. caninum han sido realizadas en explotaciones de tipo intensiva establecidas en la costa peruana; sin embargo, no se han efectuado estudios de este tipo en las zonas alto andinas donde las comunidades campesinas desarrollan una ganadería de tipo semi-extensiva y mixta (Moreno et al., 1998).

En las zonas altoandinas, el ganado pertenece mayormente a familias campesinas cuyo único sustento deriva de la crianza mixta (ganado bovino, ovino y camélidos sudamericanos) aprovechando los pastos naturales de la región. Dada la importancia de esta infección y su comprobada presencia en el país, se consideró necesario realizar el presente estudio con el objetivo de determinar la seroprevalencia de la infección por $N$. caninum en el ganado vacuno lechero criado al pastoreo de la provincia de Melgar, Puno, además de aportar datos que podrían servir de información para futuros estudios epidemiológicos relacionados a este parásito.

\section{Materiales y Métodos}

El presente trabajo se realizó entre octubre del 2001 y marzo del 2002 en la provincia de Melgar, departamento de Puno. Esta provincia tiene una altitud media de 4,000 msnm, clima seco y muy frío, con una estación seca que va de mayo a agosto y una temperatura que oscila entre los 0 a $15^{\circ} \mathrm{C}$. La precipitación pluvial media es de 250-500 $\mathrm{mm}$, donde las máximas precipitaciones ocurren en los meses de verano, esto es, entre diciembre y marzo (Moreno et al., 1998).

Se muestreó de forma aleatoria a una población bovina criada al pastoreo $(n=419)$ en siete fundos ganaderos donde predominaba el tipo de crianza mixta. Los fundos fueron elegidos por las facilidades que dieron los propietarios para la toma de muestras.

Se recolectaron muestras de sangre sin anticoagulante y el suero fue conservado a $-20{ }^{\circ} \mathrm{C}$ hasta el momento de su análisis en el Laboratorio de Parasitología de Facultad de Medicina Veterinaria de la Universidad Nacional Mayor de San Marcos. Las muestras de suero fueron diluidas en 1:200 con una 
solución salina tamponada antes de ser sometidas a la prueba de inmunofluorescencia indirecta para la detección de anticuerpos contra N. caninum.

\section{Resultados y Discusión}

Un total de 76 sueros fueron positivos a la prueba de IFI para la detección de anticuerpos específicos contra $N$. caninum, lo que se traduce en una prevalencia general de $18.1 \%$ (76/419, Cuadro 1). Resultados previos en otras zonas del país indican seroprevalencias de $43 \%$ en Cajamarca (Cabrera et al., 2000), 57\% en Arequipa (Andresen, 1999), 30\% en el valle de Lima (Silva et al., 2002) y $40 \%$ en Amazonas (Quevedo et al., 2003), de allí que se puede considerar que la seroprevalencia obtenida en Puno fue moderada.

Las prevalencias obtenidas en los siete fundos variaron desde 4.0 hasta $37.5 \%$ (Cuadro 1). Estas diferencias podrían deberse al tipo de manejo animal y a la presencia del hospedero definitivo en las zonas de pastoreo. La mayoría de los fundos en estudio practican el sistema de hatos abiertos, es decir, que introducen animales de reemplazo; y es posible que la infección se haya establecido en la zona con la llegada de animales infectados con neosporosis procedentes de zonas con prevalencias altas. La informalidad y ausencia de un control sanitario básico en la adquisición del ganado habrían facilitado el ingreso de esta infección.

La presencia del hospedero definitivo en casi todos los fundos sugiere que éste cumplió un rol importante en la propagación de la neosporosis en la zona; ya que está comprobado que la prevalencia para $N$. caninum en poblaciones bovinas que conviven con el hospedero definitivo es mayor en comparación con aquellas sin la presencia de estos (Lindsay et al., 1999; Wouda et al., 1999).

Los fundos la Raya y Santa Elena reportaron las prevalencias más bajas, y en ellos se observó una escasa y esporádica introducción de animales de reemplazo, así como la ausencia del hospedero definitivo, debido a lo inaccesible del lugar o al cumplimiento de disposiciones establecidas respecto a la presencia de canes en la zonas de pastoreo. Por otro lado, en los fundos Chuquibambilla y Macedo, que presentaron las prevalencias más altas, se comprobó una amplia presencia del hospedero definitivo en las zonas de pastoreo debido a su cercanía con centros urbanos. Además, ambos fundos introducían ganado de reemplazo con mayor frecuencia.

Se sabe que las vacas seropositivas tienen una altísima probabilidad $(95.2 \%)$ de producir descendencia infectada (Davison $e t$ al., 1999), y que la transmisión congénita sería la vía más importante para mantener la infección en un hato (Pare et al., 1996; Anderson et al., 1994, 1997). Sin embargo, las seroprevalencias obtenidas en el estudio según los grupos etáreos (Cuadro 1) no concuerdan plenamente con información de otros estudios. Además, el análisis de regresión logística determinó una relación estadística significativa entre la variables edad y lugar de procedencia con la presentación de animales seroreactores; es decir, que ambas variables estarían influyendo en la aparición de la infección. Posiblemente las evidencias epidemiológicas encontradas en el estudio no sean suficientes para asegurar que la variable edad sea verdaderamente un factor de riesgo debido a la gran diferencia en el número de animales muestreados en el estudio para cada grupo etáreo.

Lamentablemente, los resultados obtenidos no pudieron ser asociados con la presentación de abortos o infección congénita, ya que los fundos evaluados no contaban con registros que permitiesen estimar la eficiencia reproductiva.

\section{Conclusiones}

- La seroprevalencia de Neospora caninum hallada en vacunos lecheros cria- 
Cuadro 1. Seroprevalencia de $N$. caninum en bovinos criados al pastoreo en la provincia de Melgar, Puno (2002)

\begin{tabular}{cccc}
\hline & $\begin{array}{c}N^{0} \text { de animales } \\
\text { muestreados }\end{array}$ & $\mathrm{n}$ & Animales positivos \\
\cline { 3 - 4 } & & & \\
Fundos & 64 & 24 & 37.5 \\
Chuquibambilla & 93 & 19 & 20.4 \\
Macedo & 31 & 5 & 16.1 \\
Chuccamaru & 164 & 23 & 14.0 \\
Santa María & 22 & 3 & 13.6 \\
Cangalli & 20 & 1 & 5.0 \\
Santa Elena & 25 & 1 & 4.0 \\
La Raya & & & \\
Edad (años) & 171 & 36 & 21.1 \\
$1.5-2.5$ & 30 & 6 & 20.0 \\
$>2.5-3.5$ & 21 & 4 & 19.1 \\
$>3.5-4$ & 197 & 30 & 15.2 \\
\hline 4 & 419 & 76 & $18.1 \pm 3.7$ \\
\hline Total & & & \\
\hline
\end{tabular}

${ }^{1}$ Intervalo de confianza del 95\%

dos al pastoreo de la provincia de Melgar, Puno, fue moderada $(18.1 \pm 3.7 \%)$.

- La edad y el lugar de procedencia representaron factores de riesgo en la presentación de la infección, por lo que se hace necesario establecer medidas sanitarias de control y prevención a fin de evitar su incremento.

\section{Literatura Citada}

1. Anderson, M.L.; P.C. Blanchard; B.C. Barr; J.P. Dubey; R.L. Hoffman; P.A. Conrad. 1991. Neospora-like protozoan infection as a major cause of abortion in California dairy cattle. J.A.V.M.A. 198: 241- 244.
2. Anderson, M.L.; B.C. Barr; P.A. Conrad. 1994. Protozoal causes of reproductive failure in domestic ruminants.Vet. Clin. North Am. Food Anim. Pract. 10: 439-461.

3. Anderson, M.L.; J.P. Reynolds; J.D. Rowe; K.W. Sverlow; A.E. Packham; B.C. Barr; P.A. Conrad. 1997. Evidence of vertical transmission of Neospora sp. infection in dairy Cattle. JAVMA 210: 1169-1172.

4. Anderson, M.L.; A.G. Andrianarivo; P.A. Conrad. 2000. Neosporosis in cattle. Anim. Reprod. Sci. 60: 417-431.

5. Andresen, H. 1999. Neosporosis en el Perú y el mundo. Rev. Cienc. Vet. 15: 30-31. 
6. Barr, B.; J.P. Dubey; D. Lindsay; J.P. Reynolds; S.J. Wells. 1998. Neosporosis, its prevalence and economic impact. Vet. Exchange 20: 4-15.

7. Cabrera, M.; P. Ortiz; J. Claxton; D. Willians; A. Trees. 2000. Evidencia serológica de infección por Neospora caninum en el ganado vacuno del Perú. Resúmenes IV Congreso Peruano de Parasitología. Lima. p 212.

8. Conraths, F.J.; G. Schares. 1999. Diagnosis and epidemiology of Neospora caninum - associated abortions in cattle. Tierarztl. Prax. Grosstiere Nutztiere 27: 145-153.

9. Davison, H.C.; A. Otter; A.J. Trees. 1999. Estimation of vertical and horizontal transmission parameters of Neospora caninum infections in dairy cattle. Int. J. Parasitol. 29: 1683-1689.

10. Dubey, J.P. 2003. Review of Neospora caninum and neosporosis in animals. Korean J. Parasitol. 41: 1-16.

11. Lindsay, D.S.; J.P. Dubey; R.B. Duncan. 1999. Confirmation that the dog is a definitive host for Neospora caninum. Vet. Parasitol. 82: 327-333.

12. Moreno, E.; A. Canales; L. Flores; M. Pineda; D. Araníbar. 1998. Punto focal: Puno, estrategia regional para la conservación y utilización sostenible de la diversidad biológica. $\mathrm{p} 55$. Consejo Nacional del Ambiente (CONAM).
13. Paré, J; M.C. Thurmond; S.K. Hietala. 1996. Congenital Neospora caninum infection in dairy cattle and associated calfhood mortality. Can. J. Vet. Res. 60: 133-139.

14. Quevedo, J.; A. Chávez; H. Rivera; E. Casas. 2003. Neosporosis en bovinos lecheros en dos distritos de la provincia de Chachapoyas. Rev. Inv. Vet. Perú 14: 33-37.

15. Rivera, H. 2001. Etiología infecciosa del aborto bovino. Rev. Inv. Vet. Perú Supl. 1: 95-99.

16. Silva, P.; A. Chávez; H. Rivera; E. Casas. 2002. Seroprevalencia de Neospora caninum en bovinos lecheros del valle de Lima. Rev. Inv. Vet. Perú. 13: 51-55.

17. Thurmond, M.C.; S. K. Hietala. 1999. Neospora caninum infection and abortion in cattle. Am. J. Vet. Res. 58: 1381-1385.

18. Wouda, W.; T.H. Dijkstra; A.H. Kramer; C. Van Maanen; M.A. Brinkhof. 1999. Seroepidemiological evidence for a relationship between Neospora caninum infection in dog and cattle. Tijdschr. Diergeneesk. 125: 614618.

19. Wouda, W.; T. Dijkstra; A.M. Kramer; C.J. Bartels. 2000. The role of the dog in the epidemiology of neosporosis in cattle. Tijdschr. Diergeneesk. 125: 614618. 